\title{
Medidas de prevenção contra a exposição ocupacional da equipe de enfermagem aos antineoplásicos: análise crítica
}

Prevention measures against occupational exposure of the nursing staff to antineoplastic agents: critical analysis

Medidas de prevención contra la exposición ocupacional del personal de enfermería a agentes antineoplásicos: análisis critico

Silvia Maria da Silva Sant'ana Rodrigues ORCID: https://orcid.org/0000-0003-2421-8701 Centro Universitário Uninassau, Brasil

E-mail: profenf.silviasantana@gmail.com

Weber de Santana Teles ORCID: https://orcid.org/0000-0003-1770-8278 Centro de Hemoterapia de Sergipe, Brasil E-mail: arteecura@hotmail.com

Max Cruz da Silva

ORCID: https://orcid.org/0000-0002-6944-5986 Faculdade Pio Décimo, Brasil E-mail: maxlfi@hotmail.com

Ruth Cristini Torres

ORCID: https://orcid.org/0000-0002-8664-192X Instituto de Hematologia e Hemoterapia de Sergipe, Brasil E-mail: ruthcristini@gmail.com

Ana Fátima Souza Melo de Andrade ORCID: https://orcid.org/0000-0002-7024-6175 Centro Universitário Estácio de Sergipe, Brasil E-mail: anafatimamelo@hotmail.com

Marcel Vinícius Cunha Azevedo ORCID: https://orcid.org/0000-0002-5312-3333 Universidade Tiradentes, Brasil E-mail: marcelvinicius49@gmail.com

Taíssa Alice Soledade Calasans ORCID: https://orcid.org/0000-0003-0460-4437 Universidade Tiradentes, Brasil E-mail: taissa.asc@gmail.com

Ângela Maria Melo Sá Barros ORCID: https://orcid.org/0000-0003-4087-3247 Universidade Federal do Rio de Janeiro, Brasil E-mail: angelsamelo@hotmail.com

Aline Barreto Hora

ORCID: https://orcid.org/0000-0002-3930-6475 Centro Universitário Estácio de Sergipe, Brasil E-mail: aline.barretoh@ hotmail.com

Paulo Celso Curvelo Santos Junior ORCID: https://orcid.org/0000-0001-5834-6782 Universidade Tiradentes, Brasil E-mail: paulo.curvelo.jr@gmail.com Maria Hozana Santos Silva ORCID: https://orcid.org/0000-0001-5742-5366 Faculdade Ages de Medicina, Brasil E-mail: hosana_p@hotmail.com

\section{Resumo}

O presente estudo tem o objetivo de buscar na literatura cientifica evidências relacionadas às medidas eficazes de prevenção contra a exposição ocupacional da equipe de enfermagem aos antineoplásicos durante a assistência de enfermagem ao paciente oncológico. Trata-se de uma revisão de literatura das produções científicas sobre o tema indexadas, nos últimos 5 anos, nas bases de dados eletrônicas. A exposição ocupacional aos antineoplásicos, pode ocorrer em diferentes etapas, na manipulação, quando esta for realizada pelo enfermeiro, durante o transporte, a 
administração e contato com fluidos e secreções corporais até 48 horas após o termino do ciclo de quimioterapia. De forma mais generalizada, a exposição pode acontecer com a contaminação do ambiente, devido ao descarte de maneira inadequada, foram consideradas medidas eficazes de prevenção à exposição ocupacional a utilização de EPIs para os profissionais que estão na assistência direta, capacitação na área e sobre os riscos a que estão expostos e a existência de kits de derramamento nas unidades em que os procedimentos são realizados.

Palavras-chave: Antineoplásicos; Contenção de riscos biológicos; Enfermagem oncológica; Equipamento de Proteção Individual.

\begin{abstract}
This study aims to search the scientific literature for evidence related to effective preventive measures against occupational exposure of the nursing staff to antineoplastic agents during nursing care for cancer patients. This is a literature review of scientific productions on the subject indexed, in the last 5 years, in electronic databases. Occupational exposure to antineoplastics can occur at different stages, in handling, when this is performed by the nurse, during transport, administration and contact with body fluids and secretions up to 48 hours after the end of the chemotherapy cycle. More generally, exposure can occur with environmental contamination, due to improper disposal, effective measures to prevent occupational exposure were considered to be the use of PPE for professionals who are in direct care, training in the area and about risks to which they are exposed and the existence of spill kits in the units where the procedures are carried out.
\end{abstract}

Keywords: Antineoplastics; Containment of biological hazards; Oncology nursing; Individual Protection Equipment.

\title{
Resumen
}

Este estudio tiene como objetivo buscar en la literatura científica evidencia relacionada con medidas preventivas efectivas contra la exposición ocupacional del personal de enfermería a agentes antineoplásicos durante la atención de enfermería a pacientes con cáncer. Se trata de una revisión de la literatura de producciones científicas sobre el tema indexadas, en los últimos 5 años, en bases de datos electrónicas. La exposición ocupacional a antineoplásicos puede ocurrir en diferentes etapas, en la manipulación, cuando esta la realiza la enfermera, durante el transporte, administración y contacto con fluidos y secreciones corporales hasta 48 horas después de finalizado el ciclo de quimioterapia. De manera más general, la exposición puede ocurrir con contaminación ambiental, debido a una disposición inadecuada, se consideraron medidas efectivas para prevenir la exposición ocupacional como el uso de EPP para los profesionales que están en atención directa, capacitación en el área y sobre los riesgos a los que están expuestos y la existencia de kits de derrames en las unidades donde se realizan los trámites.

Palabras clave: Antineoplásticos; Contención de peligros biológicos; Enfermería Oncológica; Equipo de Protección Individual.

\section{Introdução}

O câncer, tem sido uma adversidade para a ambiência da saúde não apenas no território brasileiro, mas em todo o mundo, sendo apontada como uma das causas fundamentais de óbitos em diversos países. A prevalência da letalidade por câncer cem crescendo paulatinamente devido o envelhecimento, aumento da população e fatores de riscos. (Bray et al., 2018).

Conforme a Organização Mundial de Saúde (OMS), as malignidades neoplásicas no ano de 2018, foi responsável por cerca de 9,6 milhões de óbitos em diversos continentes. No território brasileiro é a terceiro motivo de falecimento, sendo estimado aproximadamente 600 mil casos novos durante os anos de 2018.

Regularmente, a terapêutica de escolha das neoplasias malignas é o tratamento que utiliza medicamentos para destruir as células doentes que formam um tumor denominado de quimioterapia antineoplásica, estes medicamentos possuem capacidade de toxidade para as unidades celulares que se acham em perfeita harmonia, sendo utilizados como tratamento de primeira escolha em concordância com a terapêutica de radioterapia. (Ministério da Saúde, 2018).

A enfermagem é uma das profissões mais envolvidas na assistência ao paciente oncológico, uma vez que tem participação no tratamento do paciente nas suas diferentes fases. Sendo assim, também constitui a equipe de profissionais de saúde que está mais exposto aos riscos de contaminação ocupacionais relacionados ao tratamento dos pacientes oncológicos, principalmente dos pacientes em uso de quimioterápicos antineoplásicos já que participam ativamente do processo de preparo, transporte, administração e manuseio das secreções e fluidos excretados pelos pacientes em tratamento (Sabino, Tirapelli \& Fonseca, 2015). 
Sabe-se das alterações agudas que a exposição ocupacional aos agentes antineoplásicos pode acarretar para a saúde do trabalhador a curto e longo prazo e as vias de exposição, porém, ainda não há estudos que apontem os níveis aceitáveis e seguro de exposição (Ferreira et al., 2016).

Para minimizar o risco de contaminação oriunda da manipulação e manejo com antineoplásicos, faz-se necessário orientar claramente sobre os riscos químicos e seus efeitos adversos à saúde dos trabalhadores, bem como sobre a importância das medidas de segurança apropriadas para a diminuição da exposição aos riscos ocupacionais (Oliveira, Alves \& Silva, 2013).

Logo após o profissional de enfermagem passar por capacitações, formações e adquirirem conhecimentos científicos, a compreensão dos múltiplos fatores sobre o adoecimento direciona a outros rumos recomendações e normas técnicas definidas pelos órgãos reguladores que visam a proteção da saúde do trabalhador, por meio de recomendações de boas práticas no manejo de agentes antineoplásicos (Sales, 2021).

A adesão as normas estabelecidas pelo Instituto Nacional de Câncer (INCA), Conselho Federal de Enfermagem (COFEN), agências reguladoras nacionais e internacionais, bem como a adesão as práticas de biossegurança devem ser continuamente desenvolvidas pela equipe de enfermagem e toda equipe de saúde que manuseiam quimioterápicos antineoplásicos, para que possa ter saúde plena para cuidar bem do próximo (Senna et al., 2013).

A exposição dos profissionais de enfermagem a quimioterápicos antineoplásicos podem causar diversos danos, porém, ainda se percebe o desconhecimento por parte da equipe dos profissionais quanto as medidas de prevenção contra a exposição ao risco químico oferecido pelo quimioterápicos antineoplásicos, bem como a importância de utilizá-los e aplicá-los no seu processo de trabalho diário nas unidades de oncologia (Borges, 2014).

Portanto, levando em consideração as questões supracitadas, faz-se relevante revisar as ações de prevenção a contaminação ocupacional dos profissionais de enfermagem, envolvidos diretamente na administração de quimioterápicos, bem como os fatores não inerentes a assistência que contribuem para a proteção da saúde do trabalhador envolvido no cuidado ao paciente oncológico.

O presente estudo tem o objetivo de buscar na literatura cientifica evidências relacionadas às medidas eficazes de prevenção contra a exposição ocupacional da equipe de enfermagem aos antineoplásicos durante a assistência de enfermagem ao paciente oncológico.

\section{Metodologia}

Trata-se de estudo bibliográfico, que permite ao pesquisador ter um panorama das produções cientificas relevantes a respeito do tema escolhido (Zanella, 2011). Foi realizado revisão de literatura das produções científicas sobre o tema indexadas, nos últimos 5 anos, nas bases de dados eletrônicas: Biblioteca Virtual em Saúde (BVS), Literatura Latino-americana e do Caribe em Ciências da Saúde (LILACS), PUBMED e Scientific Electronic Library Online (SCIELO), sendo utilizado os descritores: Protocolos de Quimioterapia Combinada Antineoplásica, Contenção de Riscos Biológicos, Enfermagem Oncológica, Equipamento de Proteção Individual.

É uma pesquisa do tipo exploratória, uma vez que pretende revisar as práticas de prevenção contra exposição ocupacional aplicadas durante assistência direta de enfermagem, descrever os fatores não inerentes a assistência direta que contribuem para a prevenção de exposição aos antineoplásicos, e apontar as medidas de segurança que previnem a exposição ocupacional em caso de derramamento acidental dos quimioterápicos antineoplásicos, relatados nos artigos científicos acerca do tema, com a finalidade de responder as questões norteadoras da pesquisa. Para Zanella (2011) a pesquisa exploratória é a mais adequada quando o desejo do pesquisador é aprofunda-se em um assunto pouco explorado.

Quanto à abordagem será qualitativa, uma vez que buscará interpretar os dados de maneira indutiva e interpretar como o fenômeno estudado se manifesta nas atividades do cotidiano de trabalho da equipe de enfermagem frente a assistência aos 
pacientes oncológicos. Tureto (2005), em seu artigo, cita como característica do estudo qualitativo a analise indutiva e avaliação do comportamento do fenômeno estudado, sem o intuito de mensurá-los, sendo portando a abordagem mais pertinente para este estudo.

\section{Resultados e Discussão}

\subsection{Medidas de prevenção à exposição ocupacional aos quimioterápicos}

A equipe de enfermagem é um dos grupos de profissionais que se expões a agentes físicos, químicos e biológicos, no desempenho de suas atividades. Em se tratando da assistência ao paciente oncológico, o risco químico requer muita atenção, pois constituem fator predisponente ao adoecimento de profissionais e uma ameaça a segurança em muitos ambientes laborais (Ferreira, 2016).

A exposição ocupacional aos antineoplásicos, pode ocorrer em diferentes etapas, na manipulação, quando esta for realizada pelo enfermeiro de acordo com Resolução Conselho Federal de Enfermagem 569/2018, durante o transporte, a administração e contato com fluidos e secreções corporais até 48 horas após o termino do ciclo de quimioterapia. De forma mais generalizada, a exposição pode acontecer com a contaminação do ambiente, devido ao descarte de maneira inadequada (Monteiro, 2013).

\subsection{Os quimioterápicos antineoplásicos e seus efeitos a saúde ao trabalhador}

A diferença entre quimioterápico e quimioterapia com antineoplásicos é simples: quando se tratar de um composto químico, utilizado no tratamento de doenças causadas por agentes biológicos é um quimioterápico. Quando aplicada especificamente ao tratamento do câncer, é chamada de quimioterapia antineoplásica ou antiblástica (Rodrigues, 2020).

Há uma preocupação maior com a exposição contínua ao tipo de quimioterápicos antineoplásicos em virtude dos efeitos carcinogênicos, teratogênicos e mutagênicos dessas drogas e dos efeitos deletérios a saúde do trabalhador a curto, médio e longo prazo. Principalmente, por não haver estudos, ou parâmetros nas agências reguladoras que evidenciem um nível de exposição seguro (Sagica, 2020).

As vias de entrada das substâncias no organismo são pelas vias: respiratória, cutânea e digestiva, e exercem sua ação nociva sobre os mais variados sistemas do organismo humano e na gênese das doenças profissionais dependendo da concentração e do período de exposição (Silva, 2020).

A exposição a essas drogas pode causar desde efeitos simples, como cefaleia, vertigens, tonturas, vômitos, alopecia e hiperpigmentação da pele, até efeitos mais graves com: formação de tumores e neoplasias. Além de alterações genéticas, infertilidade, aborto e malformações congênitas, disfunções menstruais e danos no DNA (ácido desoxirribonucleico) (Rocha, 2004).

\subsection{O equipamento de proteção individual e a prevenção a exposição ocupacional ao antineoplásico}

Considera-se Equipamento de Proteção Individual (EPI), todo dispositivo ou produto, de uso individual utilizado pelo trabalhador, destinado à proteção de riscos suscetíveis de ameaçar a segurança e a saúde no trabalho sendo de responsabilidade do empregador fornecer gratuitamente aos seus funcionários o EPI apropriados e em boas condições de uso, quando as medidas coletivas não oferecerem proteção total contra o risco de acidentes ou doenças relacionadas ao trabalho (Brasil, 2018).

Souza et al., (2016) conceituam o equipamento de proteção individual como medida na prevenção imprescindível a acidentes ocupacionais e para manutenção da saúde ocupacional. 
A proteção ao trabalhador durante os procedimentos envolvidos na atenção aos pacientes submetidos à quimioterapia, a adoção de medidas de proteção coletiva, como a utilização de câmaras de fluxo laminar vertical para o preparo de antineoplásicos e o uso do EPI corretamente são consideradas medidas essenciais (Rocha, 2004). (Figura 1(a) e 1(b)).

Figura 1a. Profissional paramentado para administrar QA.

Figura 1b. Cabine de fluxo laminar.
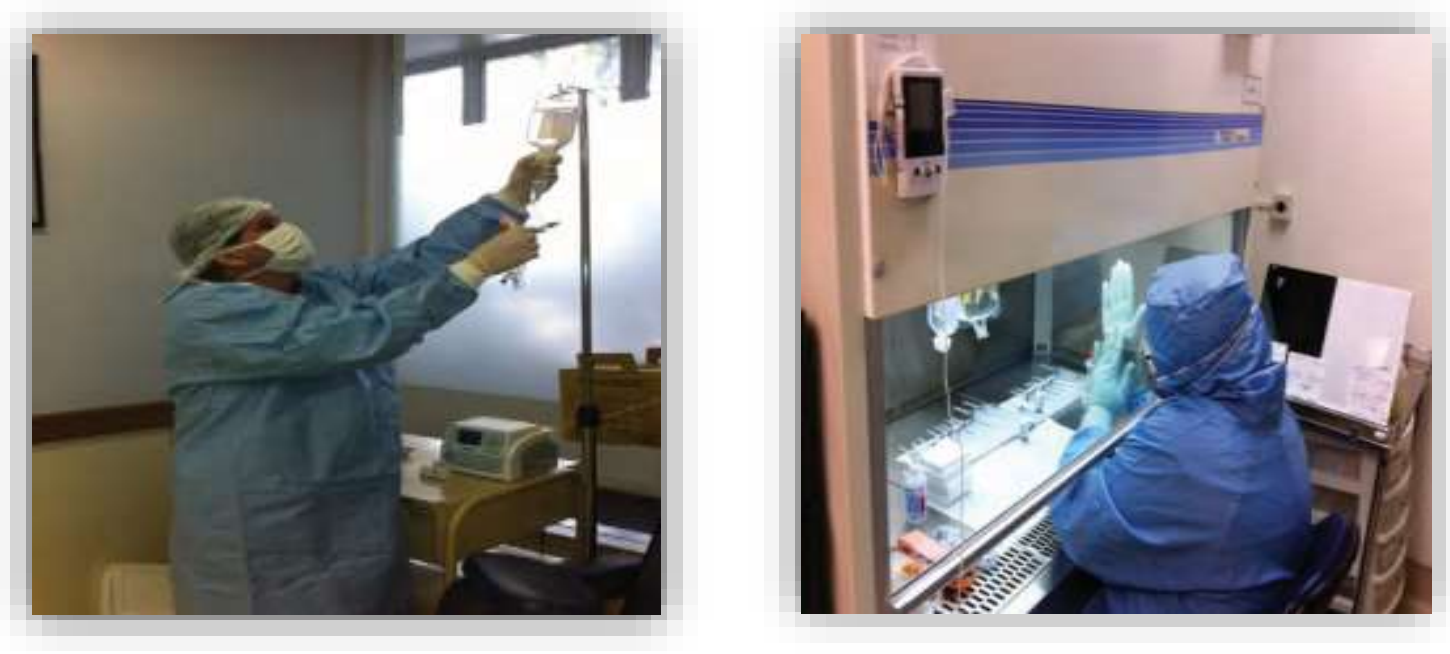

Fonte: Manual de Boas Práticas - INCA (2015).

No Brasil, a Resolução da Diretoria Colegiada no 220/2004 da Agência Nacional de Vigilância Sanitária estabelecem as condutas de manipulação, transporte, administração e o descarte do material e impõem os requisitos mínimos para o funcionamento dos Serviços de Terapia Antineoplásica (Brasil, 2004).

Existem outras legislações que normatizam e atentam para a prevenção da contaminação e exposição ao quimioterápicos antineoplásicos tanto ambiental, como dos profissionais da enfermagem, em virtude dos efeitos tóxicos e deletérios a saúde. A exemplo temos as Resoluções do Cofen 569/2018 que aprova o regulamento técnico da atuação dos profissionais de enfermagem em quimioterapia antineoplásica, a norma sobre o tratamento de resíduos sólidos do Conselho Nacional de Meio Ambiente de 05/09/1993 e a Portaria MS/GM, n 3535 de 02/09/1998, que estabelece critérios para cadastramento de centros de atendimento de oncologia (Brasil, 1998).

As utilização de equipamento de proteção individual como forma de prevenção adequada e eficiente contra a exposição ocupacional ao risco químico dos antineoplásicos durante a assistência de enfermagem ao paciente oncológico, principalmente na fase da administração com a paramentação completa, bem como a utilização do EPI durante a assistência direta e manuseio de excretas e fluídos corporais do paciente em uso de antineoplásicos, após 48 horas do fim do ciclo de quimioterapia, conforme recomendação da RDC 220/2004, minimizam o risco de contaminação por esse fármaco.

A utilização de equipamentos de proteção individual é uma das medidas utilizadas pelos profissionais de enfermagem para se protegerem dos riscos advindos dessa medicação e que os profissionais reconhecem que a não adesão às normas estabelecidas podem acarretar muitos riscos e possíveis agravos a sua saúde (Senna, 2013).

Para a administração de quimioterápico as agências reguladoras nacionais e internacionais recomendam a utilização de luvas de procedimento, máscara de carvão ativado, avental impermeável, touca, óculos.

É de suma importância utilização do EPI pelos profissionais de saúde, o fornecimento de treinamento para a utilização do equipamento de proteção de maneira correta e adequada para que a prevenção à exposição ocupacional seja eficaz (Martins, 
2015). Os pesquisados deste estudo evidenciaram que o uso de tal medida de proteção deve estender-se por até 48 horas após a infusão da quimioterapia, mostrando conhecimento das recomendações da RDC n ${ }^{\circ}$ 220/2004 da ANVISA (Brasil, 2004).

Conforme agência norte-americana Occupational Safety and Health Administration - OSHA, os EPIs que devem ser utilizados na manipulação ou administração de quimioterápicos são: luvas de látex descartáveis e sem talco; aventais descartáveis, com mangas longas, fechados na parte frontal, punhos com elásticos e com baixa permeabilidade; máscaras, de preferência com carvão ativado e óculos de proteção (OSHA, 2016).

No Brasil a recomendação que a RDC n ${ }^{\circ} 220 / 04$ informa é que sejam utilizados pelo menos durante a manipulação de fluidos e excretas, luvas de procedimento e avental impermeável de manga longa. No entanto, cabe ao senso crítico do profissional, verificar a necessidade de utilizar outros EPI complementares como os óculos, touca e máscara de carvão ativado (Brasil, 2004).

Os EPIs estabelecidos pelas agências reguladoras nacionais e internacionais, estão relacionadas as possíveis vias de contaminação com o antineoplásico que são: vias aéreas por conta dos aerossóis, oral por ingestão de alimentos, pele e mucosa.

Os enfermeiros que lidam com antineoplásicos devem ter conhecimento sobre as medidas de biossegurança para prevenir a exposição ocupacional, saber sobre os padrões existentes e saber como utilizar os equipamentos de proteção individual para que ocorra a proteção efetiva à exposição ao risco químico (Ferreira et al., 2016).

É necessário que a equipe de enfermagem que assiste ao paciente com câncer, principalmente na fase de tratamento com quimioterapia, tenha conhecimento dos riscos a que está exposto, sobre as medidas de proteção existentes e como utilizálas a seu favor, para que ocorra a prevenção eficaz da exposição.

\subsection{O papel da educação continuada na prevenção da exposição ocupacional}

A educação permanente consiste na potencialização do desenvolvimento pessoal e promove tanto capacitação técnica específica dos sujeitos, como a aquisição de novos conhecimentos, conceitos e atitudes (Paschoal, Mantovani \& Méier, 2007).

As ações de educação em serviço têm papel na promoção da informação, agregam elementos que qualificam a conduta e os comportamentos inadequados, e com isso levem a transformação de hábitos predeterminados por processos de trabalho defasados à medida que o trabalhador se observa integrado ao processo de trabalho ao qual está inserido (Loro \& Zeitoune, 2007).

Dentro da vertente da educação permanente, encontra-se a educação em serviço que acontece no ambiente de trabalho visando melhoria da qualidade das ações de saúde desenvolvida pelos profissionais da instituição.

Neves et al., (2011) inferiram em seu estudo sobre Segurança dos trabalhadores de enfermagem e fatores determinantes para adesão aos equipamentos de proteção individual, entre outros fatores, a falta de educação permanente alertando para a percepção dos profissionais quanto aos riscos em que estão expostos, contribuem para a baixa adesão ao uso de equipamentos de proteção individual.

Monteiro, Chaves \& Figueiredo, (2013) concluem em sua pesquisa sobre a importância da educação continuada e permanente, como estratégia que possibilite a enfermagem como forma de garantir a segurança da equipe, e que repercutirá na melhoria da qualidade dos serviços prestados e na segurança e saúde ocupacional.

Dentre os fatores não relacionados a assistência direta ao paciente em uso de quimioterapia e que contribuem para prevenção à exposição ocupacional durante a assistência de enfermagem a educação continuada.

Silva et al., (2015) em seu estudo verificaram que 90\% da amostra de profissionais de enfermagem estudados não recebeu nenhum treinamento específico para desenvolverem suas atividades na unidade de quimioterapia, tal achado vai de encontro ao que normatiza a NR 32 e a Resolução da Diretoria Colegiada RDC N 220/2004, que trazem o entendimento de que o profissional capacitado e treinando, sabe reconhecer as situações de risco e se prevenir da exposição ocupacional. 
A conscientização através de capacitação é uma ferramenta de grande valor para promoção e proteção da saúde dos trabalhadores, já que capacitação dos profissionais proporciona a prevenção de acidentes e leva a melhoria dos processos de trabalho, devendo aquela ser realizada pelas próprias instituições através de programas de educação permanente (Oliveira, 2013).

Um outro fator que contribuem para a prevenção a exposição ao risco químico oferecido pelos quimioterápicos é existência de protocolos quem padronizem e orientem aos profissionais envolvidos as práticas de assistência com o paciente oncológico, garantindo dessa forma a continuidade da assistência e promoção da saúde do trabalhador.

Ferreira et al., (2016) ao estudarem as medidas de biossegurança na administração de quimioterapia antineoplásica, verificaram que os enfermeiros estudados relatavam em seus discursos sobre a importância e a necessidade de existência de protocolos escritos para orientar quanto ao manejo.

O estudo acima reforça ainda mais a ideia de que a criação de protocolos é uma medida indireta de prevenção a exposição, uma vez que normatiza quanto aos procedimentos e norteia quanto ao tipo de ação em situações de intercorrências com esses agentes.

Oliveira et al., (2013) encontraram como resultado de sua revisão de literatura sobre o tema, que a elaboração e implementação de protocolos como sendo uma dificuldade e que isto se deve ao fato de que o enfermeiro responsável pela sua elaboração está envolvido com outras atividades assistências e burocráticas.

As organizações internacionais, como a National Institute for Occupational Safety and Health (NIOSH) e a Occupational Health and Safety Administration (OSHA), dentre outras medidas de segurança que visam a reduzir a exposição dos profissionais ao risco químico, com intuito de minimizá-lo, recomendam protocolos para o manuseio das drogas.

A medida que os profissionais entendem os riscos e os danos causados pela exposição ao risco químico a curto, médio e longo prazo, a elaboração e implementação poderia ser priorizada, desta forma a educação em saúde e capacitação como forma de atualização dos conhecimentos do profissional, demonstram sua relevância.

Desta forma, entende-se que o profissional, quando capacitado quanto aos riscos a que está exposto, treinados em como utilizar o EPI de maneira correta, minimizam a exposição ocupacional ao risco que o antineoplásicos oferece. Segundo a NR 32, cabe ao empregador promover os treinamentos necessários para assegurar a segurança da saúde do trabalhador, bem como a instituição de protocolos e normas operacionais que padronizem todo procedimento de manuseio e descarte dos quimioterápico em sua unidade, visando à prevenção à exposição.

Embora existam outros motivos que contribuem para a baixa adesão as medidas de prevenção contra a exposição ao risco químico, a educação permanente e a padronização dos procedimentos contribuem para a sensibilização dos profissionais envolvidos para a importância do cumprimento das medidas instituídas no ambiente laboral.

\subsection{Kit de derramamento e seu papel como medida de prevenção a exposição ocupacional aos quimioterápicos antineoplásicos}

A Resolução da Diretoria Colegiada - RDC nº 220, de 21 de setembro de 2004, cita em seu anexo sobre biossegurança, a necessidade de os serviços de terapia antineoplásica manterem um kit de derramamento para os casos de extravasamento acidental no ambiente.

A finalidade do kit de derramamento é de contenção e limpeza primária do antineoplásico extravasado de forma acidental, o qual devido ao risco químico que confere, deve ter um manejo e descarte adequado considerando o que preconiza a resolução n 306 de gerenciamento de resíduos de saúde. Embora sua principal função seja evitar a contaminação do ambiente, ele também funciona como medida de prevenção a exposição dos trabalhadores. 
Pela RDC - n 220/2004 o kit deve ser composto por no mínimo, luvas de procedimentos, avental de baixa permeabilidade, compressas absorventes, proteção respiratória, proteção ocular, sabão, descrição do procedimento e o formulário para o registro do acidente, recipiente identificado para recolhimento dos resíduos. (Figura 2).

Figura 2. Kit derramamento.

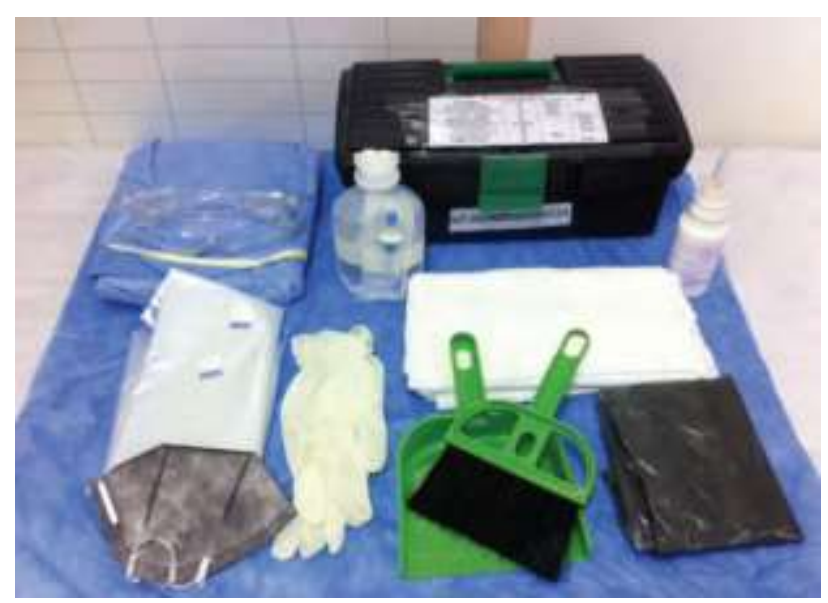

Fonte: Manual de Boas Práticas - INCA (2015).

Embora seja pré-requisito para o funcionamento adequado dos serviços de terapia antineoplásica, sabe-se que ainda tem instituições que não o possuem, ou quando tem não sabem como utilizá-lo, devido à falta de capacitação (Sousa, 2016).

Quanto as medidas de prevenção à exposição em caso de derramamento acidental de antineoplásicos, a literatura aponta para a existência de um kit de derramamento nas unidades para as situações em que ocorrer o extravasamento e evitar a contaminação ambiental (Gonçalves, 2021).

Nos casos de derramamento acidental de antineoplásicos faz-se necessário a tomada de determinadas precauções afim de evitar a contaminação ambiental e exposição dos trabalhadores, e que visando tais finalidades para iniciar a limpeza da área de derramamento deve haver a paramentação, após identificação, restrição de acesso, e delimitação da área do derrame com compressas absorventes (Oliveira, 2013).

Acerca dos itens necessários nos casos de derramamento de antineoplásicos, o kit de derramamento é necessário e sua ausência, além de ser uma não conformidade com a recomendações da ANVISA, pode comprometer a saúde e a segurança do profissional que manuseia o antineoplásico, pois em casos de derramamento acidental, não saberiam como atuar e como proceder para conter o derramamento (Ferreira, 2016).

O kit de derramamento é citado em $25 \%$ dos artigos utilizados em seu estudo, e que isso é devido ao fato que ele minimiza o risco de exposição ao agente químico nos casos de derramamento, evento de ocorrência comum durante a administração de medicações (Oliveira, 2013).

Portanto, faz-se necessário a existência de kits de derramamento acidental nas unidades em que estejam sendo realizados os quimioterápicos, bem como o treinamento de uso adequado, para que este possa cumprir com o papel de prevenção de contaminação do ambiente e consequentemente prevenção de contaminação da equipe envolvida (Gonçalves, 2021).

\subsection{O enfermeiro oncologista e as medidas de prevenção à exposição ocupacional ao risco químico dos antineoplásicos}

O enfermeiro oncologista exerce papel importante na prevenção à exposição aos antineoplásicos, uma vez que tem formação especifica e sabe reconhecer os riscos a que a equipe está exposta, entende das causas e efeitos do contato com esse tipo de medicamento. 
A atuação do enfermeiro na educação permanente com os profissionais de enfermagem, é imprescindível para evitar possíveis danos que possam comprometer a saúde e a capacidade laboral do trabalhador (Senna, 2013).

Oliveira, (2013) atribuiu o processo de educação continuada e permanente da equipe de enfermagem ao enfermeiro, assim como a formulação de protocolos de assistência de enfermagem e, também, a garantia de uso deles. Os autores entendem que o enfermeiro especialista tem competência para priorizar e ressaltar os cuidados específicos à administração de quimioterapia, desta forma garantindo a prevenção exposição ocupacional.

É papel do enfermeiro, viabilizar aos seus liderados o acesso institucional a educação continuada que irá promover a transformação da prática, e sensibilização para a construção e promoção de atitude éticas e seguras (Baroni, 2013).

O enfermeiro oncologista enquanto líder de equipe e especialista, deve treinar, capacitar e orientar sua equipe de modo a prestar assistência necessária e de acordo com as necessidade e cuidados que o paciente oncológico demanda, bem como é responsável por zelar pela prevenção a exposição ocupacional oferecido pelos quimioterápicos antineoplásicos, por meio da instituição e padronização da assistência por meio de protocolos, prestação de orientação e treinamento quanto ao uso de EPIs e como proceder em casos de derramamento acidental do quimioterápico (Borges, 2014).

\section{Considerações Finais}

A utilização dos equipamentos de proteção individual, nas diferentes fases da assistência direta ao paciente em ciclo de quimioterapia antineoplásica é primordial, para a segurança da saúde do trabalhador, tendo em vista as consequências que a exposição a esses fármacos pode acarretar.

A literatura aponta o EPI como medida eficaz na prevenção à exposição ocupacional ao quimioterápico antineoplásicos quando utilizado de forma correta e nas diferentes etapas da assistência ao paciente até 48 horas após o ciclo de quimioterapia.

É primordial, que as informações acerca dos riscos e medidas de prevenção, sejam discutidas, pesquisadas e difundidas. Sejam promovidas pelos núcleos de educação continuada das instituições que oferecem tratamento a pacientes oncológicos, como forma de prevenção a exposição e padronização das medidas preventivas, visando minimizar os efeitos da exposição que os quimioterápicos antineoplásicos acarretam.

Nas unidades oncológicas deve-se manter o kit de derramamento para a prevenção da contaminação ambiental e da equipe envolvida, em caso de derramamento acidental, e junto com ele a equipe deve ser treinada em como utilizá-lo.

Um outro ponto discutido foi sobre o papel do enfermeiro oncologistas, enquanto líder de equipe, consciente dos riscos que a terapia antineoplásicos pode trazer a equipe, como promotor das medidas eficazes de prevenção.

Desta forma, pode-se afirmar que os objetivos específicos deste trabalho foram alcançados, assim como o objetivo geral, uma vez que reuniu as principais evidencias de prevenção a exposição aos antineoplásicos encontrados na literatura nacional e internacional.

Espera-se que este trabalho contribua com a sensibilização dos profissionais da saúde sobre a importância de utilizar as medidas de prevenção existente e que desperte em outros pesquisadores o interesse sobre o assunto já que ainda existem poucas publicações voltadas para o tema de modo tão específico.

\section{Referências}

ANVISA. Agencia Nacional De Vigilância Sanitária. (2004). Resolução da diretoria colegiada- RDC $n^{o} 220$, de 21 de setembro de 2004. Dispõe sobre o regulamento técnico de funcionamento dos Serviços de terapia antineoplásica.

Baroni, F. C. A. L. (2013). O trabalhador de enfermagem frente ao gerenciamento de resíduo químico em unidade de quimioterapia antineoplásica. Rev Min Enferm. 17(3), 554-559.

Brasil. (2018). Resolução COFEN 568 de 19 de Fevereiro de 2018. Dispõe sobre o regulamento técnico da atuação dos profissionais de enfermagem em quimioterapia antineoplásica, nos termos do anexo desta resolução. Diário oficial da União, Brasília. 
Brasil. (1998). Portaria n 3535 de 02 de setembro de 1998. Estabelece critérios para o cadastramento de centros de atendimento em oncologia. Diário Oficial da União, Brasília, 02 de setembro de 1998.

Brasil. (1978). Ministério do Trabalho e Emprego. Portaria $\mathrm{n}^{\circ}$ 3214, de 8 de junho de 1978. Altera Norma Regulamentadora nº -Equipamento de Proteção Individual - EPI Diário Oficial da República Federativa do Brasil, Brasília (DF).

Brasil. (1998). Ministério do Trabalho e Emprego. Portaria no 19, de 9 de abril de 1998. Altera Norma Regulamentadora n 7 - Programa de Controle Médico de Saúde Ocupacional. Diário Oficial da República Federativa do Brasil, Brasília (DF), 1998.

Bray, F. et al. (2018). Global Cancer Statistics. GLOBOCAN estimates of incidence and mortality worldwide for 36 cancers in 185 countries. CA: a cancer journal for clinicians, Hoboken, 68(6), 394-424.

Brasil. (2008). Ministério do Trabalho e Emprego. Portaria no 939, de 19 de novembro de 2008. Altera Norma Regulamentadora no 32 Segurança e Saúde nos Trabalhos em serviços de Saúde. Diário Oficial da República Federativa do Brasil, Brasília, 19 de novembro de 2008.

Borges, G. G. (2014). Biossegurança na central de quimioterapia: o enfermeiro frente ao risco químico. Rev Bras Cancerol. 60(3), 247-50.

Costa, L. C. C, Franco, R. E. L. (2009). Central de quimioterapia: aspectos básicos de gerenciamento.: Portal Farmacêutico.

Gonçalves, D. S., Silva, H. C. S., \& Conceição, R. (2021). Extravasamento de antineoplásicos em Centro Oncológico. Pesquisa, Sociedade e Desenvolvimento, 10(11), e361101119814.

Gurgel, I. O., Sá, P. M., Reis, I. F. \& Mattia, A.L. (2018). Prevalência de práticas integrativas e complementares em pacientes submetidos à quimioterapia antineoplásica. Cogitare enferm.

Ferreira, A. R. (2016). Medidas de Biossegurança na Administração de Quimioterapia Antineoplásica: Conhecimento dos Enfermeiros. Revista Brasileira de Cancerologia. 62(2), 137-145.

Instituto Nacional de Câncer José Alencar Gomes da Silva [INCA]. (2020). Estimativa 2020: incidência de câncer no Brasil. Coordenação de Prevenção e Vigilância: Rio de Janeiro.

Loro, M. M., \& Zeitoune, R. C. G. (2017). Estratégia coletiva de enfrentamento dos riscos ocupacionais de uma equipe de enfermagem. Rev. esc. enferm. USP, 51(1), e03205.

Manual de boas práticas exposição ao risco químico na central de quimioterapia: conceitos e deveres ministério da saúde. (2015). Instituto Nacional de Câncer José Alencar Gomes da Silva (INCA).

Martins, D. (2015). Manipulação de quimioterápicos pelos profissionais da saúde. Rev. Ibirapuera, 12(10), 57-61.

Monteiro, A. B. C. (1999). Manuseio e preparo de quimioterápicos: Uma colaboração ao processo reflexivo da conduta da enfermagem. Rev. latino-am. Enfermagem. 7(5), 127-135.

Monteiro, L. A., Chaves, D. D. C., Figueiredo, L. F. (2013). Conhecimento dos profissionais de enfermagem sobre a ciclofosfamida em um hospital universitário. Revista Eletrônica de Enfermagem, 15(2), 430-6.

Ministério da Saúde (BR). (2019). Sistema de Informações hospitalares do SUS. Ministério da Saúde.

Neves, H. C. C. (2011). La seguridad de los trabajadores de enfermería y los factores determinantes para adhesión a los equipamientos de protección individual.Rev. Latino-Am. Enfermagem, 19(2), 354-361.

National Institute For Occupational Safety And Health - NIOSH (2004). Alert: preventing occupational exposure to antineoplastic and other hazardous drugs in health caresettings. Cincinnati: National Institute for Occupational Safety and Health.

Oliveira, A. D. S. (2013). Riscos ocupacionais da exposição da equipe de enfermagem a quimioterápicos: revisão integrativa de literatura. Revista de enfermagem UFPE online. Recife. 7(1), 794-802.

Paschoal, A. S., Mantovani, M. F., \& Meier, M. J. (2007). Percepção da educação permanente, continuada e em serviço para enfermeiros de um hospital de ensino. Rev. esc. enferm. USP, São Paulo, 41(3), 478-484.

Rocha, F. L. R., Marziale, M. H. P., \& Robazzi, M. L. C. C. (2004). Perigos potenciais a que estão expostos os trabalhadores de enfermagem na manipulação de quimioterápicos antineoplásicos: conhecê-los para prevení-los. Rev. latino- am. Enfermagem. Ribeirão Preto. 12(3), 511-517.

Rodrigues, H. S. (2020). Perfil de interações medicamentosas de agentes antineoplásicos orais (OAAs) dispensados para pacientes oncológicos. Pesquisa, Sociedade e Desenvolvimento. 9(8), e145985369.

Sabino, B., Tirapelli, B., \& Fonseca, S.M. (2015). Biossegurança em Enfermagem Oncológica: Uma Revisão Integrativa. Revista Recien, $5(13), 29$ - 43.

Sagica, T. P. (2020). Spill and extravasation of antineoplastic chemotherapics in a university hospital. Research, Society and Development, 9(7), e552974320.

Sales, C. L. C., et al., (2021). Contribuições e dificuldades da equipe de enfermagem na implementação de cuidados paliativos ao paciente oncológico. Research, Society and Development, 10(3), e30410312460.

Senna, M. H. (2013). Segurança do trabalhador na manipulação de antineoplásicos. Revista Avances en Enfermería. 31(1), 14-158.

Silva, L. S., \& Cavalcanti, V. (2012). Riscos quimicos hospitalares e gerenciamento dos agravos à saúde do trabalhador. Revista de Pesquisa: Cuidado é Fundamental Online. 1(12), 21-24. 
Research, Society and Development, v. 10, n. 15, e88101522708, 2021

(CC BY 4.0) | ISSN 2525-3409 | DOI: http://dx.doi.org/10.33448/rsd-v10i15.22708

Silva, H. R. (2020). Terapia personalizada em oncologia, considerando farmacogenética e antineoplásicos atuais. Pesquisa, Sociedade e Desenvolvimento. 9(7), e631974312.

Sousa, A. F. L. (2016). Representações sociais da Enfermagem sobre biossegurança: saúde ocupacional e o cuidar prevencionista. Rev. Bras. Enferm. Brasília. 69(5), 864-871.

Souza, C. B. (2015). Antineoplásicos e os riscos ocupacionais para os enfermeiros: uma revisão integrativa. Enfermeria Global. 40(1), 311-325.

Turato, E. R. (2005). Métodos qualitativos e quantitativos na área da saúde: definições, diferenças e seus objetos de pesquisa. Rev. Saúde Pública, São Paulo. 39(3), 507-514.

United States Departamento Of Labor. (2016). Occupational Health And Safety Administration. Controlling Occupational Exposure To Hazardous Drugs. In: OSHA tecnical manual. US Departamento of Labor.

Zanella, L. C. H. (2011). Metodologia de pesquisa. (2a ed.), Departamento de Ciências e Administração/UFSC. 